\title{
Developing Effective Interuniversity Partnerships and Community-Based Research to Address Health Disparities
}

\author{
Timothy S. Carey, MD, MPH [director], Daniel L. Howard, PhD [director of sponsored \\ programs], Moses Goldmon, EdD [associate professor], James T. Roberson, PhD [dean], \\ Paul A. Godley, MD, PhD, MPP [professor of medicine], and Alice Ammerman, DrPH, RD \\ [director] \\ Cecil G. Sheps Center for Health Services Research, University of North Carolina at Chapel Hill, \\ Chapel Hill, North Carolina.
}

Shaw University, Raleigh, North Carolina.

Shaw University Divinity School, Raleigh, North Carolina.

Shaw University Divinity School, Raleigh, North Carolina.

University of North Carolina at Chapel Hill, Chapel Hill, North Carolina.

Center for Health Promotion and Disease Prevention, University of North Carolina, Chapel Hill, North Carolina.

\begin{abstract}
Health disparities are an enormous challenge to American society. Addressing these disparities is a priority for U.S. society and especially for institutions of higher learning, with their threefold mission of education, service, and research. Collaboration across multiple intellectual disciplines will be critical as universities address health disparities. In addition, universities must collaborate with communities, with state partners, and with each other. Development of these collaborations must be sensitive to the history and unique characteristics of each academic institution and population. The authors describe the challenges of all three types of collaboration, but primarily focus on collaboration between research-intensive universities and historically black colleges and universities.

The authors describe a four-year collaboration between Shaw University and the University of North Carolina at Chapel Hill (UNC-CH). These universities strategically developed multiple research initiatives to address health disparities, building on modest early success and personal relationships. These activities included participation by Shaw faculty in faculty development activities, multiple collaborative pilot studies, and joint participation in securing grants from the Agency for Health care Research and Quality of the federal Department of Health and Human Services and the National Institutes of Health, including a P-60 Project EXPORT center grant. These multiple activities were sometimes led by UNC-CH, sometimes by Shaw University. Open discussion of problems as they arose, realistic expectations, and mutual recognition of the strengths of each institution and its faculty have been critical in achieving successful collaboration to date.
\end{abstract}

Although America's population is among the healthiest in the world, substantial problems exist with health and health care in the United States. The health status of the U.S. population is characterized by marked disparities in health care and health outcomes among major ethnic and income groups. ${ }^{1}$ For instance, health outcomes across multiple areas, such as cancer,

Correspondence should be addressed to Dr. Carey, CB 7590, Cecil G. Sheps Center for Health Services Research, University of North Carolina at Chapel Hill, Chapel Hill, NC 27599; telephone: (919) 966-7100; fax: (919) 966-8918; 〈tim_carey@ unc.edu〉.. 
perinatal outcomes, and cardiovascular care, are substantially worse among U.S. blacks and Latinos than among whites. ${ }^{2-5}$ The causes of these observed health disparities are still incompletely understood, but appear to be multiple and complex, ${ }^{6}$ including interacting elements from both medical practice and social factors. Public health data indicate that health outcomes vary substantially for different populations, while recent clinical research indicates that the medical care received by minority groups is also unequal to that of whites. That is, individuals from minority ethnic groups or low-income groups are less likely than are whites or high-income groups to receive appropriate, evidence-based, quality treatment for cardiac disease, cancer, preventive care, and multiple other health concerns. ${ }^{7}$ Two recent federal reports, Unequal Treatment, by the Institute of Medicine and the National Health Disparities Report, by the Agency for Health care Research and Quality (AHRQ) of the federal Department of Health and Human Services, have confirmed that these disparities in health care access and delivery are persistent. ${ }^{8,9}$

As interdisciplinary research has become much more common over the past decade, interdisciplinary approaches have emerged as a potential strategy to effectively address the multiple and complex challenges posed by health disparities. ${ }^{10}$ Health disparities may arise from economic issues, environmental issues including endemic violence and lack of recreational facilities in minority areas, chronic stress from poverty and racism, or discrepancies in care provided by the health care system. Addressing these issues requires expertise in multiple social science fields (economics, environmental science, sociology, and so forth) in addition to clinical medicine. The National Institutes of Health (NIH) has recently launched a research "roadmap initiative" specifically designed to foster interdisciplinary research, and especially various types of translational research. ${ }^{11}$

Much of the discussion regarding translational research within the scientific community has focused on "bench to bedside" translation: How can researchers and physicians more rapidly and efficiently transfer basic scientific discoveries in genetics, cell biology, pathophysiology, and pharmacology into clinical applications? Less discussed, but just as important to the nation's health, is the translation of effective clinical and health promotion interventions to reach populations at risk. The knowledge gained through health disparities research is particularly appropriate to this type of "bedside to community" translational research. If an effective clinical intervention, such as colorectal cancer screening, is applied disproportionately to only one privileged segment of the population, then health disparities based on ethnicity or income may persist or even widen in the future as novel diagnostic and therapeutic applications come into clinical use. Similarly, if effective behavior modification interventions such as diet and exercise are not implemented in a culturally sensitive manner by known and trusted individuals, associations, and institutions, researchers and educators may continue to experience difficulty in collaborating with diverse populations in implementing lifestyle changes that can contribute a great deal to the elimination of health disparities. Researchers, clinicians, educators, and policymakers must carefully attend to this second, implementation-type of research translation in order to begin improving the health status of racial and ethnic minority and socioeconomically disadvantaged groups.

A second theme of the NIH roadmap initiative is the development of new fields of research and scholarship, representing a "melding" of existing disciplines. ${ }^{12}$ An often-cited example is the intense collaboration among the disciplines of genetics, cell biology, biochemistry, anatomy, and some types of physiology. While degrees are still individually awarded in these constituent disciplines, individuals from the various disciplines are increasingly working together in complementary ways to reach a common goal. Multiple neuroscience disciplines have also grown closer. ${ }^{13}$ 


\section{Health Disparities Studies}

We propose that health disparities studies may be undergoing a similar melding of disciplines. Scientists across disciplines who are interested in the problems of disparities in the financing, organization, delivery, and outcomes of care, as well as community educational interventions, are increasingly working together to conduct and translate research findings that increase the likelihood of effectively assisting hard-to-reach populations. Through these collaborations, each discipline brings its own perspective to bear on a common problem. A partial list of disciplines involved in collaborative health disparities research and educational interventions at our own institutions includes clinical medicine, epidemiology, health policy, health economics, biostatistics, nutrition, health behavior/health education, practical theology, sociology, medical anthropology, psychology, and environmental science. Each constituent field brings a different but interrelated perspective to the study of health disparities. The melding of these various perspectives increases the likelihood of identifying transferable solutions to the multiple and complex challenges that contribute to health disparities. Of particular importance for health disparities research is translating research findings into action; researchers and society alike perceive a sense or urgency around these issues. ${ }^{14}$ We may now be approaching the time when we can begin to attach a label of "health disparities studies" to such endeavors. This label emphasizes the common goal across disciplines and will aid communication among researchers and policymakers.

Yet at the present time, we do not favor the establishment of separate academic departments of health disparities studies or degrees in health disparities studies. While such steps would highlight the importance of addressing health disparities and fostering the evolving discipline, unanticipated consequences might result from such formalization. If universities begin to offer degrees in health disparities studies, departments of medicine, sociology, or epidemiology might begin to feel absolved from addressing health disparities in their coursework or research agendas. Separate departments of health disparities studies might even marginalize the field at exactly the time that many disciplines need to bring cutting-edge theory and practice to these persistent problems. We believe that units that encourage interdepartmental and interdisciplinary work, such as programs or centers targeted at addressing health disparities, are more appropriate mechanisms to spread the growing knowledge of health disparities studies. The University of North Carolina at Chapel Hill (UNC-CH) recently established a certificate program in health disparities studies. Students enrolled in any one of a number of graduate programs, such as nursing, pharmacy, or public health, take several courses from an approved list in order to obtain the certificate, which is earned in addition to a master's or doctoral degree.

Over the past five years the federal government, along with various foundations and advocacy groups, has made a concerted effort to more forcefully address the longstanding health disparities in the U.S. Universities are assuming a substantial role in these initiatives. Medical schools and other health profession schools have a twofold obligation in these initiatives: they are sites where new health care knowledge is generated, and they embrace the service mission to care for vulnerable populations. In addition, universities of all types exist within definable communities that can be valuable partners in developing solutions to health care disparities. These communities include the surrounding geographic areas and the demographic groups who seek care at university hospitals and clinics. Institutions that serve ethnic and income minorities have longstanding and close relationships with both the geographic and demographic communities they serve. The histories of these minority-serving institutions are varied, but many originated during the era of de jure segregation in education in the United States. These historically black colleges and universities (HBCUs) have, in the modern era, maintained as part of their mission the aim of reaching those who otherwise might not have higher education opportunities. In $2000,40 \%$ of black students receiving a degree in a biological science 
graduated from HBCUs. ${ }^{15}$ Some of these schools are also mission-specific in preparing their graduates to serve the minority communities in which they are based.

Below, we describe a collaborative health disparities research effort between Shaw University (a historically black liberal arts university) and UNC-CH (a research-intensive institution) that focuses on the elimination of health disparities between racial and ethnic minority and majority populations through community-focused research. Although single investigators can conduct successful health disparities research studies, the multiple and complex nature of health disparities makes collaboration a particularly well-suited approach to such research. The merging of multiple disciplines across universities with complimentary histories, missions, and priorities fosters a greater understanding of the many complex factors that contribute to health disparities. Partnerships such as the collaboration between Shaw and UNC-CH also make available a plethora of resources that can help to address the specific interests of populations bearing a disproportionate burden of poor health outcomes.

Universities can and should be involved in three overall types of collaboration: with their communities, with state and local governments, and with other universities. We will discuss the first two types of collaboration only briefly. Our discussion will center on our interuniversity collaboration experiences focusing on community-based research. As representatives of both UNC-CH and Shaw, we have built upon a history of collaboration between administrators and faculty members across the two institutions to form the CarolinaShaw Partnership for the Elimination of Health Disparities. We will first describe the types of university collaborations, and then focus on the progress and challenges we encountered in our interinstitutional collaboration from 2000 to 2005.

\section{University-Community Collaboration}

Historically, much research has appropriately focused on intervention and analysis at the level of the individual. Yet more recent studies have considered the community as a distinct entity with definite and unique characteristics, as well as varying structures and leadership. In ideal circumstances, the community can function alongside the university as both a research subject and a collaborator. ${ }^{16}$ While "community structure" is usually associated with discussions regarding minority populations, all populations have communities, leaders, and good and bad ways of interacting. These structures affect how populations are accessed for research in general as well as for community-based participatory research. One particularly exciting example is collaborations between universities and black churches. ${ }^{17}$ The church has a particularly important role in the black community, with a widely recognized focus on health issues as an integral component of the pastoral mission.

The field of community-based participatory research (CBPR) has been instrumental in the area of health research for some decades. ${ }^{18}$ The overall tenets of CBPR are well established. ${ }^{19}$ Involving the community early in the research process at the level of assisting in formulating culturally sensitive research methods-from question formulation and data collection through interpretation of results- have been found to be productive, albeit time-intensive. A precondition for expansion of CBPR methods will be improved and more widespread training in CBPR methods, as well as in grant proposal and article review, for the academics and communities collaborating with each other. ${ }^{20}$

\section{University Collaboration with State and Local Organizations}

The mission of the university is threefold: education, research, and service. While service is an important component, it is secondary to education. Similarly, research is a priority of many higher educational institutions, but certainly not all. Some institutions have a primarily educational mission and conduct little research. State governments and their constituent 
organizations, in contrast, are primarily service oriented and not focused on developing new knowledge through research. In addition, state organizations do not have a substantial professional training mission. Collaborations between universities and state organizations would seem to be natural, since they have complimentary, rather than competitive, goals. Both universities and state government are large, complex organizations. These organizations may appear opaque to those who do not work within them. Although their bureaucracies are different, both academics and state employees are familiar with their own administrative structures, and are likely to be less frustrated when encountering a new bureaucracy in their research partner. The UNC-CH/Shaw Partnership works with the North Carolina Office of Minority Health as part of our overall initiative, participating in one another's conferences and seminars. We have found that the community contacts of the academic and state organizations are different and complement each other.

\section{Collaborations between Universities}

While to an external observer it may appear that all academic institutions are similar, each collaborating partner has its own culture that must be learned by the other for complex collaboration to succeed. When a new faculty member comes to a university, even from a similar research institution, our experience has been that it may take 12 to 18 months to learn the institutional culture of the new facility. In collaborations across different universities, it will likely take at least as long for participants to learn each other's institutional cultures. Issues such as the administrative structure, reward structure, internal compensation for teaching and service, and tenure and promotion criteria are important yet widely variable components of institutional culture. When a predominantly research-based university and a teaching-intensive institution address either service or research issues together, issues of translation and collaboration will be complex. Specifically, faculty incentives to perform even similar activities may be quite different at each institution. A teaching-intensive institution may value and reward the number of courses taught, teaching awards, and number of students advised.

Research-intensive institutions may value publications, dollar value of grants brought in, and percentage of salary covered by external funds. All faculty wish to develop and succeed, but the criteria for success may differ between institutions. Similar to other types of collaboration discussed above, these successes generally occur at the level of the individual. That is, individual meetings, research mentoring relationships with faculty and students, collaborative writing of manuscripts, and so forth are all appropriate evidence of successful collaborative relationships. Each university should define in advance what it is seeking from each collaboration with another institution, so that success can be recognized when it occurs.

Multiple federal initiatives have set goals for developing improved research infrastructure at HBCUs. The majority of the 105 HBCUs are located in the southeastern United States, and most are over 100 years old. HBCUs enrolled $14 \%$ of all black students in higher education in 1999 , although they constitute only $2 \%$ of the country's 4,182 institutions of higher education. HBCUs awarded $24 \%$ of all baccalaureate degrees earned by African Americans nationwide. ${ }^{21}$ Since 2002, the White House Initiative on Historically Black Colleges and Universities was designed to provide a structured effort to help HBCUs to access federally funded programs. 22 Thirty federal agencies participate in the initiative under Executive Order 13256, which includes annual goals and plans for entering into appropriate grants, contracts, or cooperative agreements with HBCUs across a variety of research and educational projects. ${ }^{23}$ These initiatives have occurred with varying success in individual cases, although overall the HBCUs involved are becoming more research-based over time. Recent initiatives such as the EXCEED (EXcellence Centers to Eliminate Ethnic/racial Disparities) Initiative of the AHRQ and Project EXPORT (Centers of EXcellence in Partnerships for community Outreach, Research in health disparities and Training) of the NIH have explicitly encouraged relationships between researchintensive universities and HBCUs. ${ }^{24}$ While investigators at majority institutions have 
periodically worked with faculty at HBCUs, the potential presence of substantial grant awards provides a significant incentive to additional activity. These financial incentives may be sufficient to lead to collaboration between research-intensive and minority institutions, but certainly in no way assure a successful collaboration.

\section{Collaboration between UNC-CH and Shaw University}

Beginning in 2000, the University of North Carolina and Shaw University have built a significant portfolio of mutual educational and research endeavors. UNC-CH is a large, research-intensive state university based in Chapel Hill, North Carolina, with $\$ 500$ million in external research grant funding each year. UNC-CH houses five health science schools (medicine, public health, pharmacy, nursing, social work) on the Chapel Hill campus. UNC$\mathrm{CH}$ was a segregated institution until the 1950s. Shaw University, founded in 1865 , is the oldest historically black college in the South and houses graduate schools of divinity and education. Shaw also has a history of involvement in health education: in the late 19th and early 20th centuries, the university housed the Leonard School of Medicine, the nation's first four-year black medical school. ${ }^{25}$ Shaw has extensive ties with black churches in the southeastern United States, having trained generations of ministers.

While the relationship between the two institutions has always been collegial, the recent availability of significant potential grant funding provided a substantial incentive to develop closer relations. However, the presence of these grant funds, while necessary to conducting significant research, does not ensure a successful collaboration. Faculty at UNC-CH began working with faculty at Shaw under the aegis of Daniel Howard, a faculty member at Shaw who had previously completed a postdoctoral research fellowship at UNC-CH's Cecil G. Sheps Center for Health Services Research. This preexisting personal and professional relationship provided mutual understanding of goals as well as an initial basis of trust on which to develop future relationships. In addition, the dean of the divinity school at Shaw, the late Dr. Joseph C. Paige, had provided consultation to several UNC-CH projects that worked with minority communities. Dr Paige provided critical advice in forging the relationships and in providing introductions across schools. Although these early experiences were modest in both their scope and their financial impact on both institutions, their success reduced the perceived risk of the subsequent, more substantial initiatives. Dean J. T. Roberson of the Shaw University Divinity School built on the work of Dr. Paige in developing a vision for enhanced links between Shaw and multiple churches throughout North Carolina.

The collaboration between the universities started relatively modestly. UNC-CH applied for and received an EXCEED program project grant from the AHRQ, while a modest subcontract to Shaw assisted in the conduct of secondary data analyses to evaluate the role of continuity of care for elderly black and white North Carolinians. This proposal provided Dr. Howard with a lead role on one component of the project, and allowed the multiple UNC-CH faculty working under the project grant to get to know Shaw and its faculty. Meanwhile, students from Shaw worked in internships at UNC-CH. These internships involved participation of the students in the research process, as well as exposure to seminars and other activities at UNC-CH.

Identifying activities appropriate for undergraduate students required several modifications of expectations and activities. This initial, modest relationship was perceived as successful, and both institutions were thus open to additional, incremental collaboration. This allowed what was in essence a "bootstrapping" approach, using the grant resources and working relationships of the initial activities to build additional projects and grant applications. Expected challenges of grants administration at Shaw included the necessity of using the UNC-CH institutional review board (IRB) for human subjects certifications, and limited grants management capacity. In addition, Shaw University, a teaching-intensive institution, had little experience with shifting job descriptions when a faculty member spent an increasing proportion of his or her 
time doing research, with no policies in place on how to reduce teaching loads as grant funding rose.

As the collaborative endeavor progressed, faculty at UNC-CH then assisted Shaw faculty with securing a health services research infrastructure grant (M-RISP) from the AHRQ. This infrastructure grant was based at Shaw with a subcontract to UNC-CH. This type of symmetry, in which grants were either primarily based at the research institution and subcontracted to the minority institution or vice versa, established mutual trust and a series of intertwined platforms on which to build additional initiatives. All parties were cognizant of the limited grant management research infrastructure at Shaw, and several early applications were specifically designed to enhance data management, grant management, and administrative infrastructure. These included a three-year process to develop an independent IRB at Shaw, as well as on-site data management capacity. The availability of infrastructure grants to support these activities was critical in Shaw's rapid research expansion. Had the university had to rely solely on facilities and administrative (or "indirect") funds to support such infrastructure development, the progress would have been much slower. An eventual challenge to the institution will be making the transition away from the infrastructure development funds as Shaw becomes an autonomous initiator of research.

The most substantive collaborative project to date has been funded by a Project EXPORT center five-year grant on health disparities. Receipt of the center grant solidified the relationship between Shaw and UNC-CH, while its duration provided time to develop infrastructure and collaborative relationships with less continuous pressure to raise funds. The collaboration was codified with a name, the Carolina-Shaw Partnership for the Elimination of Health Disparities, and goal statements that included training young investigators in research methods in health disparities, collaboration between the universities on major community-based research projects, and enhancing Shaw University's capacity to conduct independent research. This series of EXPORT center grants, administered by the National Center for Minority Health and Health Disparities, encouraged linked grants across majority and minority institutions. Through this mechanism, UNC-CH and Shaw have parallel budgets, thus avoiding negotiating subcontracts and obviating concerns regarding the distribution of financial and administrative (indirect) funds between the primary and subcontracting institutions.

The aim of the Project EXPORT center grant was in part to develop the universities' research infrastructures. Another significant aim was addressing health disparities through community engagement. Shaw is developing a network with black churches throughout North Carolina. The long-term goal of the Shaw Divinity School is to engage the congregations of these churches in a two-way dialogue centered on health. Shaw, in collaboration with UNC-CH and the North Carolina Office of Minority Health, will provide health information to the churches to be shared with the congregations. The congregations will tell Shaw and its collaborators their health concerns and indicate their interest in joint research projects on health disparities. As of 2005, 25 churches have been recruited, computers are being installed in the churches, and information is being collected regarding the communities' health concerns.

In order to foster this interuniversity research and collaboration, UNC-CH faculty have conducted seminars at Shaw and have mentored junior faculty. These topics have included systematic literature review, the roles of pilot studies, and a variety of methods issues such as ambulatory care sensitive conditions. Shaw faculty have led similar seminars and conference workshops at UNC-CH. Shaw has begun hiring both junior and senior faculty with active research experience in order to "jump start" its research programs. At the operational level, administrators and business personnel from UNC-CH have been working with Shaw on issues relating to grants administration. The amount of health disparities research at UNC-CH has grown to the point that the unit housing many of the grants, the Cecil G. Sheps Center for 
Health Services Research, has organized a Program on Health Disparities. This program allows sharing of staff across several projects, resulting in enhanced efficiency. In addition, UNC-CH has established a program on Ethnicity, Culture and Health Outcomes (the ECHO program), which facilitates and coordinates multiple educational and research initiatives. The ECHO program has been particularly effective in communicating with multiple HBCUs around the state, emphasizing stepwise, progressive collaboration, and minimizing faculty or staff overcommitment, which may lead to feelings of disappointment or lack of support regarding collaboration.

\section{Negotiating Potential Communication Issues}

The relationship between UNC-CH and Shaw continues to mature but, as expected, is also going through growing pains. Working relationships between different types of institutions sometimes result in misunderstanding, confusion, and delay despite the best of intentions. For instance, research-intensive institutions have, in the past, had relationships with smaller universities that involved either poorly thought out subcontracts or inadequate sharing of academic "credit" in terms of presentations and authorship. Similarly, universities communicating with minority communities have sometimes lacked the appropriate cultural sensitivity or have inadequately compensated and communicated with research subjects. ${ }^{26}$ Recognizing these past issues and attempting avoid them is an important and ongoing component of developing collaborative projects. Faculty sometimes assume that since their intentions are good they do not need to acknowledge and address past collaboration problems. Yet doing so is important and does not imply a faculty member's personal responsibility for past issues, but is critical to understanding and working within the appropriate social and historic context. Below, we will describe four issues requiring open communication that collaborators at UNC-CH and Shaw have encountered over the past several years: data ownership, authorship issues, expectations regarding mentoring, and negotiating the home administrative unit for new initiatives at UNC-CH and Shaw.

An early example of an issue requiring careful resolution involved data ownership. Initial collaborative research projects such as focus groups and baseline surveys required data storage, analysis, and archiving. Early on, faculty at Shaw expressed concerns regarding "ownership" of data. This involved both intellectual ownership issues (e.g., who would perform secondary analyses, who would be an author of the subsequent publications) and the physical location of the data files. Faculty at UNC-CH felt somewhat defensive about the assumption that they might misinterpret focus-group data, and were quite concerned that the overall research endeavor was being slowed down. An additional concern related to a feeling of responsibility felt by faculty at Shaw for the data collected from study participants they had personally recruited. This led to important discussions regarding the necessity of basing recruitment on a full disclosure of study requirements, benefits, and potential harms rather than solely on the reputation of the recruiter. The problem was satisfactorily addressed by a small interinstitutional committee that developed a statement of data "stewardship," thus avoiding the more emotionally loaded term "ownership," and established a multi-institution group to address secondary data analyses. If such analyses were performed, the interinstitutional committee would review the results and make sure the co-investigators were reflective of the origin of the data and the partnership between UNC-CH and Shaw.

A similar interinstitutional group addressed the issue of authorship, assuring that both institutions would be represented in publications arising out of the partnership. A clear procedure was developed so that faculty from both UNC-CH and Shaw would have the opportunity to author such publications. A logo was developed incorporating both institutions' seals and stationary was printed, although resistance to sharing logos was substantial at some bureaucratic levels. 
A third issue involved communication about the manner of collaboration among faculty at the two universities. We noted that faculty at both institutions used three different descriptive terms almost interchangeably: consultation, collaboration, and mentoring. Our view is that these three relationships between investigators are quite different, and the consequences of miscommunication regarding these terms may be substantial.

Consultation is the communication of a question from a primary investigator to another "consulting" individual in the hopes of reaching an answer, often regarding a very specific issue within an overall research project. The skills sought from the consultant may range from biostatistical advice to clinical consultation. Consultants may not be involved in writing the research proposal, do not necessarily take intellectual responsibility for the conclusions of the entire work, and may not be involved with the project throughout its entire course. Consultants respond when called, but will not be proactive in seeking out work on a project.

Collaboration generally refers to the relationship between co-investigators. Collaborators are involved with the project through its course, understand (but may not contribute to) the entire scope of the project, and generally are coauthors of resulting publications. Collaborators attend meetings and conference calls, and are very much involved in two-way collegial communication with the project leader.

Mentoring is a very different relationship from either consulting or collaboration. A mentor may not be the project leader, but is generally a senior investigator who gives advice to the project leader or co-investigator throughout the course of the project. This advice is usually expected to go beyond technical input on research issues, and involve guidance on project management, career development, and progress of the research ideas from one study to the next. Mentoring may continue beyond a single project.

Given these differences, clarity about the nature of faculty involvement in collaboration is crucial. If an investigator at a research university thinks she or he is going to be a consultant advising on technical issues, such as utilization of antihypertensive medications within a secondary data analysis, but the minority institution views the relationship as one of mentoring, the potential for misunderstanding is obvious. A year into the project, each faculty member may still be waiting for the other to call and initiate dialogue. Faculty at UNC-CH and Shaw have learned to be explicit with each other regarding the anticipated nature of collaborative relationships, recognizing that these relationships can, of course, change over time.

A final issue has been the maturing relationship between UNC and Shaw. Previous relationships were essentially solely between Shaw and UNC-CH and largely administered through one UNC administrative unit, the Cecil G. Sheps Center for Health Services Research. These relationships have broadened to include other units and campuses. These relationships have generally thrived, with increasing levels of comfort among faculty, administrators, and staff. The number of active grants at Shaw has grown substantially, and has grown beyond health services research. Shaw is now establishing research relationships with other units on the UNC-CH campus, as well as with other major research universities. These new initiatives may have administrative "homes" at various departments on the UNC-CH campus, as well as with other research-intensive universities. Sheps Center administrators and staff will need to recognize this expansion as an indicator of success, not a rejection of previous collaborative relationships. Shaw faculty and administration have found that there are clear advantages to collaborating with multiple research units, including the availability of more faculty and access to funding streams that a health service research unit may not otherwise be able to access. However, the time and cost of developing each new collaborative relationship will likely lead to modest development, not exponential growth. 


\section{Conclusions}

Research is becoming increasingly collaborative across faculty and institutions. Health disparities research, drawing upon clinical medicine, epidemiology, social science, and economics, is an ideal example of the advantages of interdisciplinary research. Health disparities studies is a novel field that reflects the translation of clinical and social science to populations and communities. Interuniversity collaboration adds an additional level of complexity to this translational research. Such collaboration is certainly possible, but when very diverse institutions work together, the need for leadership structure, early and frank discussion of potential areas of conflict, and setting of clear benchmarks for progress becomes all the more important. Recent federal and foundation initiatives to encourage health disparities research have yielded initial success. In order to sustain this success, ongoing effort and nurturing will be essential. Health disparities research is a timely and important field that represents an area of translational research falling well within the NIH roadmap initiative. Partnerships between research-intensive and teaching-intensive, minority-serving universities can draw on the strengths of both institutions, leading to enhanced knowledge and improved health for Americans.

\section{Acknowledgments}

Thanks to Ms. Chanetta Washington for careful review of and comments on the manuscript.

This project was supported by Agency for Health care Research and Quality Grant P01 HS 10861 and National Center on Minority Health and Health Disparities Grants P60 MD244-01 and P60 MD239-01.

\section{References}

1. Agency for Healthcare Research and Quality. 2004 National Healthcare Disparities Report. AHRQ Publication No 05-0014 Dec 04

2. Gornick ME, Eggers PW, Reilly TW, et al. Effects of race and income on mortality and use of services among Medicare beneficiaries. N Engl J Med 1996;335:791-99. [PubMed: 8703185]

3. Wong MD, Shapiro MF, Boscardin WJ, Ettner SL. Contribution of major diseases to disparities in mortality. N Engl J Med 2002;347:1585-92. [PubMed: 12432046]

4. Hajat A, Lucas JB, Kington R. Health outcomes among Hispanic subgroups: data from the National Health Interview Survey, 1992-95. Adv Data 2000;310:1-14. [PubMed: 10977762]

5. Buescher PA. A review of available data on the health of the Latino population in North Carolina. $\mathrm{N}$ C Med J 2003;64:97-105. [PubMed: 12854303]

6. Cooper LA, Hill MN, Powe NR. Designing and evaluating interventions to eliminate racial and ethnic disparities in health care. J Gen Intern Med 2002;17:477-86. [PubMed: 12133164]

7. Siegel S, Moy E, Burstin H. Assessing the nation's progress toward elimination of disparities in healthcare: the national healthcare disparities report. J Gen Intern Med 2004;19:195-200. [PubMed: 15009799]

8. Smedley, BD.; Stith, AY.; Nelson, AR., editors. Unequal Treatment: Confronting Racial and Ethnic Disparities in Health Care. National Academy Press; Washington, DC: 2003.

9. National Healthcare Disparities Report: Summary. Agency for Healthcare Research and Quality; Rockville, MD: Feb2004 [28 July 2005]. 〈http://www.ahrq.gov/qual/nhdr03/nhdrsum03.htm〉.

10. Epstein AM, Ayanian JZ. Racial disparities in medical care. N Engl J Med 2001;344:1471-73. [PubMed: 11346815]

11. Zerhouni E. The NIH roadmap. Science 2003;302:63-65. [PubMed: 14526066]

12. National Institutes of Health. Training for a new interdisciplinary research workforce.. NIH guide. [28 July 2005]. 〈http://grants1.nih.gov/grants/guide/rfa-files/RFA-RM-04-015.html〉.

13. Arnold SE. Bedside to bench and back again: translational neuroscience research and geriatric psychiatry. Am J Geriatr Psychiatry 2004;12:122-25. [PubMed: 15010339] 
14. Lavizzo-Mourey R, Knickman J. Racial disparities, the need for research and action. N Engl J Med 2003;349:1379-80. [PubMed: 14523147]

15. Suitts S. Fuelling education reform: historically black colleges are meeting a national science imperative. Cell Biol Educ 2003;2:205-6. [PubMed: 14673481]

16. Israel BA, Schultz AJ, Parker EA, Becker AB. Community-based participatory research: policy recommendations for promoting a partnership approach in health research. Educ Health 2001;14:182-97.

17. Ammerman A, Corbie-Smith G, St. George DM, et al. Research expectations among African American church leaders in the PRAISE! Project: a randomized trial guided by community based participatory research. Am J Public Health 2003;93:1720-27. [PubMed: 14534228]

18. Community-Based Participatory Research: Assessing the Evidence. File Inventory, Evidence Report/ Technology Assessment Number 99. Agency for Healthcare Research and Quality; Rockville, MD: [28 July 2005]. AHRQ Publication No. 04-E022-2. 〈http://www.ahrq.gov/clinic/cbprinv.htm〉.

19. The Church as a "Natural" Partner in Health Promotion. Jackson County Health Advisory Council, Jackson County, Florida. Principles of Community Engagement/CDC/ATSDR Committee on Community Centers for Disease Control/ATSDR Committee on Community Engagement. Centers for Disease Control and Prevention (US), Public Health Practice Program Office; Atlanta, GA: 1997.

20. Leung MW, Yen IH, Minkler M. Community based participatory research: a promising approach for increasing epidemiology's relevance in the $21^{\text {st }}$ century. Int J Epidemiol 2004;33:499-506. [PubMed: 15155709]

21. Snyder, TD.; Hoffman, CM. Digest of Education Statistics, 2001. Department of Education, National Center for Education Statistics; Washington, DC: May. 2002 NCES Publication No. 2002130US.

22. White House Initiative on Historically Black Colleges and Universities. U.S. Department of Education; Washington, DC: Aug. 2002 ERIC Publication No. ED468353.

23. Federal Register. Executive Order 13256, Thursday, February 14, 2002.

24. Stryer DB, Weinick RM, Clancy CM. Reducing racial and ethnic disparities in health care. Health Serv Res 2002;37:xv-xxiii. [PubMed: 12479487]

25. Savitt, TL. The education of black physicians at Shaw University, 1882-1918.. In: Crow, JJ.; Hatley, FJ., editors. Black Americans in North Carolina and the South. Vol. 1984. The University of North Carolina Press; Chapel Hill: p. 161-88.

26. Washington AE, Napoles-Springer A, Forte DA, Alexander M, Perez-Stabile E. Establishing centers to address treatment effectiveness in diverse ethnic groups: the MEDTEP experience. Ethnicity Health 2002:7731-42. 